$2^{\mathrm{a}}$ PARTE

COMENTARIO AL ENSAYO DE UNA NUEVA EXPOSICIÓN DE LA DOCTRINA DE LA CIENCIA DE FICHTE 



\title{
NECESIDAD, LIBERTAD Y FILOSOFÍA EN \\ FICHTE. UNA LECTURA DE LA PRIMERA \\ INTRODUCCIÓN A LA DOCTRINA DE LA \\ CIENCIA DE $1797^{1}$
}

\section{NECESSITY, FREEDOM AND PHILOSOPHY IN \\ FICHTE. A READING OF THE FIRST \\ INTRODUCTION TO THE DOCTRINE OF \\ SCIENCE OF 1797}

\author{
MARÍA JiMENA SOLÉ \\ $U B A / C O N I C E T$
}

Puede decirse que tanto la Primera Introducción a la Doctrina de la Ciencia, así como la Segunda Introducción y el Ensayo de una nueva exposición de la Doctrina de la Ciencia - textos publicados por Fichte en el Philosophisches Journal entre febrero de 1797 y marzo de 1798 - tienen su origen, al igual que la Doctrina de la Ciencia nova methodo, en la insatisfacción de su autor frente a ciertos aspectos de la anterior exposición de su sistema. Efectivamente, tanto el Fundamento de toda la Doctrina de la Ciencia como Sobre el concepto de la Doctrina de la Ciencia habían generado gran perplejidad y fuertes críticas entre el público lector. Así pues, podría sospecharse que es a partir de esta primera recepción negativa de su sistema que Fichte se vuelve consciente, entre otras cosas, de la necesidad de preparar a sus lectores para ingresar a la DC y, por lo tanto, de la importancia de anteponer una introducción.

\footnotetext{
${ }^{1}$ Quisiera agradecer aquí los comentarios y sugerencias del Dr. Jacinto Rivera de Rosales que han servido para mejorar mi escrito, así como los de los integrantes del Grupo de investigación «Fichte entre la Ilustración y el Romanticismo», que coordino desde 2010 junto con Mariano Gaudio en la Facultad de Filosofía y Letras de la Universidad de Buenos Aires.
} 
En efecto, es notable que en el Prólogo que Fichte publica en la segunda entrega del Fundamento..., a principios de 1795, afirma que esas palabras preliminares hubiesen sido innecesarias si este texto no hubiese llegado a manos de un público al que no estaba destinado, un público que, por no haber estado presente en sus lecciones, no había logrado comprender su pensamiento y se había apresurado a proferir críticas, objeciones y burlas. ${ }^{2}$ Poco después, sin embargo, cuando Fichte retoma la enseñanza de la DC en las aulas de la Universidad de Jena recurriendo a un nuevo método y paralelamente se dispone a publicar una nueva versión de su sistema por entregas en el Philosophisches Journal, decide ofrecer no una, sino dos introducciones. La primera - tanto la publicada en la revista como la que inaugura el manuscrito de Krause de la DC nova methodo- aborda una cuestión que se revela como fundamental y en la que, puede suponerse, autor y lector, maestro y alumno, deben poder ponerse de acuerdo antes de ingresar al sistema propiamente dicho. Se trata del problema, tan antiguo como fundamental, de determinar qué es la filosofía.

Ahora bien, al recorrer la Primera Introducción a la DC de 1797, nos vemos inclinados a pensar que este texto no cumple meramente la función de preparar al público lector para un conjunto de ideas que su autor considera novedoso y revolucionario mediante la exposición del concepto de filosofía que se manejará a lo largo de la obra. Creemos que esta Primera Introducción y los temas allí abordados son en realidad una necesidad impuesta por el mismo sistema. Fichte ha tomado conciencia de que su pensamiento será resistido por muchos e inaccesible para otros, porque la filosofía — la DC — requiere de sus lectores u oyentes no tanto un determinado nivel de comprensión teórica o un consenso respecto del uso de ciertos términos, sino la adopción de una determinada actitud práctica. La Primera Introducción es, pues, el resultado de haber comprendido que la filosofía, tal como Fichte la entiende, implica un problema central, que ella misma debe explicitar: el problema de cómo se transmite una filosofía y, por consiguiente, el problema de quién es su destinatario.

Estas son, pues, las cuestiones que, según creemos, animan el desarrollo de la Primera Introducción a la DC y cuya complejidad y significación pretende poner en evidencia la lectura que ofrecemos a continuación. Su complejidad reside,

\footnotetext{
${ }^{2}$ Cf. GA I/2, 251 y ss.
} 
según veremos, en el hecho de que parece haber cierta circularidad en el planteo fichteano: para acceder a la DC se requiere ya ser, en cierta medida, un filósofo tal como este sistema lo entiende. Su significación reside, tal como creemos y esperamos poder exponer en las próximas páginas, en que la concepción fichteana de la filosofía se revela en este texto como el punto de partida necesario desde el cual se construye todo el sistema.

\section{1.}

Antes de ingresar propiamente en el texto que nos ocupa, examinemos brevemente los textos anteriores a 1797 en los que Fichte se propone introducir su sistema, con el fin de encontrar ya allí algún esbozo de los problemas que aquí nos interesan.

Antes de su llegada a Jena, Fichte redacta Sobre el concepto de la Doctrina de la ciencia o de la asi llamada filosofía, que apareció en mayo de 1794. El Prefacio a este texto se ocupa principalmente de exponer la intención de su autor: brindar una exposición de los lineamientos básicos de su sistema, para que los estudiantes de la Universidad de Jena juzgaran por sí mismos «si debían confiarse a su dirección en el camino de la primera entre las ciencias" ${ }^{3}$. Sin embargo, Fichte da muestras ya de cierta preocupación en cuanto a la posibilidad de transmitir su doctrina, pues afirma que es consciente de que pasarán varios años hasta que su pensamiento adquiera una forma acabada. ${ }^{4}$ En este sentido, solicita a sus lectores que le hagan la justicia de no rechazar su sistema antes de haberlo examinado en su totalidad y agradece a los amigos dispuestos a ofrecerle críticas constructivas. Pero también se anticipa y se dirige a aquellos que se encuentran inclinados a poner en ridículo las esperanzas de su ciencia. A ellos, Fichte les pide que contengan su risa hasta que su empresa esté "formalmente fracasada y abandonada»" $y$ concluye:

${ }^{3}$ GA I/2, 110. Versión castellana: Fichte, J.G., Sobre el concepto de la Doctrina de la Ciencia, trad. Bernabé Navarro, Centro de Estudios Filosóficos, Universidad Nacional Autónoma de México, 1963 , p. 10.

${ }^{4}$ Cf. GA I/2, 110.

${ }^{5}$ GA I/2, 111. Versión castellana: Fichte, J.G., Sobre el concepto de la Doctrina de la Ciencia, trad. Bernabé Navarro, Centro de Estudios Filosóficos, Universidad Nacional Autónoma de México, 1963, p. 12. 
Que se burlen entonces ellos de nuestra confianza en la humanidad, a la que ellos mismos pertenecen, $y$ de nuestras esperanzas en las grandes aptitudes de la misma; que repitan entonces su sentencia consolatoria: la humanidad es algo que ya no tiene remedio; así fue y será siempre — cuantas veces necesiten de consuelo. ${ }^{6}$

De modo que, aún si Fichte sabía que al ofrecer una presentación todavía provisoria de su DC se exponía a las burlas y las críticas de personas malintencionadas, no tiene reparos en declarar su confianza en la humanidad y en que ésta será apta para recibir y valorar su descubrimiento filosófico. Pero, por otro lado, la finalidad de este texto — que los estudiantes puedan decidir si están interesados en asistir a sus clases o no- - refleja ya cierta expectativa por parte de Fichte respecto de su auditorio: él desea que sus oyentes se encuentren prevenidos acerca de su posición filosófica y que decidan libremente si quieren ser instruidos en ella o no.

Pocos meses más tarde, en septiembre de 1794, apareció la primera entrega del Fundamento de toda la DC que, tal como adelantamos, no presenta ningún texto introductorio sino que se inicia con la exposición del primer principio ${ }^{7}$. Fueron precisamente las vituperaciones, tergiversaciones y calumnias proferidas contra este texto, lo que motivó a Fichte a incluir, junto con la segunda entrega

${ }^{6}$ GA I/2, 111. Versión castellana: Fichte, J.G., Sobre el concepto de la Doctrina de la Ciencia, trad. Bernabé Navarro, Centro de Estudios Filosóficos, Universidad Nacional Autónoma de México, 1963, p. 12.

${ }^{7}$ Como se sabe, este texto es el resultado de la recopilación de los apuntes que Fichte redactó para el uso de los asistentes a los cursos que dictó durante los dos primeros semestres de su actividad como profesor en la Universidad de Jena. Durante el semestre de verano de 1794, Fichte dictó un curso sobre la DC y en septiembre de ese año sus apuntes, originalmente escritos exclusivamente para los oyentes de sus lecciones, aparecieron publicados por la casa editorial Gabler (partes I y II). El semestre siguiente, Fichte se ocupó de la parte práctica de su sistema y hacia mediados de 1795, los apuntes que distribuía entre sus oyentes se encontraban también a disposición del público (parte III) junto con un Prefacio a toda la obra. Ese mismo año Gabler editó una versión conjunta de ambas partes, bajo el título de Fundamento de toda la Doctrina de la ciencia como manuscrito para sus oyentes. No es, pues, difícil comprender que esta primera exposición del sistema, que presenta todas las desprolijidades e imperfecciones propias de un texto que fue escrito bajo la presión de la imprenta y que contiene una doctrina que aún no estaba del todo madura, haya generado gran perplejidad y duras críticas entre el público lector. Fichte mismo llegó pronto a la conclusión de que redactar apuntes para los oyentes, a medida que avanzaba el semestre, había sido una mala idea (véase la carta de Fichte a Weisshuhn de julio de 1794 y la carta a Goethe del 30 de septiembre de 1794). 
del libro publicada a principios de 1795, un Prólogo que, tal como adelantamos, él mismo considera que hubiera sido innecesario en una situación diferente ${ }^{8}$. Sin embargo es en este texto donde se esbozan algunas de las ideas contenidas en la Primera Introducción a la DC. De modo que ciertas reflexiones que en 1795 son motivadas por el contexto inmediato de recepción de su obra y que Fichte mismo considera superfluo en relación con el sistema, dos años más tarde reaparecen como la introducción a la DC.

En principio, Fichte intenta desvelar las causas de las críticas recibidas. Afirma en este Prólogo que el Fundamento de toda la DC no estaba destinado al público, pues se trataba de una exposición extremadamente imperfecta y defectuosa y señala diferentes motivos por los que esto es así. En primer lugar, remite al hecho de que los Fundamentos... son la compilación de las notas que había ido redactando y publicando a medida que dictaba su curso en la Universidad, con el fin de que los estudiantes tuvieran de antemano el escrito cuando se iba a dar esa lección. De este modo, el lector carecía de la instancia de la exposición oral, que permitía una iluminación de los pasajes oscuros o las dificultades que el texto podía dejar abiertas $^{9}$. En segundo lugar, Fichte admite que su decisión consciente de evitar una terminología fija - la cual, según él, es lo que contribuye a transformar los sistemas en esqueletos secos - contribuye asimismo a complicar la comprensión de su pensamiento $^{10}$. Finalmente, Fichte aduce un motivo de otra índole:

Particularmente estimo necesario recordar que no pretendía decirlo todo, sino dejar también algo para que mis lectores pensaran. Hay muchos malentendidos que preveo con seguridad, y que habría podido remediar con un par de palabras. Sin embargo, tampoco he dicho este par de palabras, porque podría oprimir el pensamiento personal. La Doctrina de la ciencia no debe imponerse en absoluto, sino que debe ser una necesidad, como lo era para su autor. ${ }^{11}$

Así pues, si bien el primer motivo que explica la oscuridad de esta obra —el hecho de que el lector carece de la instancia de explicación oral— es accidental

${ }^{8}$ Cf. GA, I/2, 251.

${ }^{9}$ Cf. GA, I/2, 251.

${ }^{10}$ Cf. GA, I/2, 251.

${ }^{11}$ GA, I/2, 253. Versión castellana: J.G. Fichte, Doctrina de la ciencia, trad. e intr. Juan Cruz Cruz, Aguilar, Buenos Aires, 1975, p. 9. Énfasis nuestro. 
al texto y a su contenido, tanto el segundo como el tercero - la decisión consciente de evitar una terminología fija y la decisión de no decirlo todo- parecen ser requisitos impuestos por el carácter de la DC misma. En efecto, Fichte sostiene que algunas oscuridades del texto, que podrían haber sido subsanadas fácilmente - quizás con una explicación, quizás recurriendo a un lenguaje técnico específico - tienen una función y un sentido: el de obligar a sus lectores a ejercer lo que Fichte denomina Selbstdenken: a pensar por sí mismos. Esto es así porque, según sostiene explícitamente, su sistema no debe ser impuesto sino que los lectores deben sentir que la filosofía es para ellos una necesidad ${ }^{12}$.

Sin embargo Fichte no acepta todas las críticas recibidas. Respecto de aquellos que dicen encontrar incomprensible su pensamiento — expuesto ya en el Fundamento... ya en Sobre el concepto...- escribe:

Si esa obra es el límite de su comprensión, entonces ella es el límite de mi inteligibilidad; nuestros espíritus están separados entre sí por ese límite, y yo les rogaría que no perdieran su tiempo leyendo mis obras. Si esta nocomprensión tiene un fundamento, cualquiera que sea, hay en la Doctrina de la Ciencia misma un fundamento por el cual ella tiene que permanecer siempre incomprensible para ciertos lectores; este fundamento consiste en que ella supone la facultad de libertad de la intuición interna [das Vermögen der Freiheit der inneren Anschaunng]. ${ }^{13}$

Poniendo en suspenso la confianza en la humanidad toda que había expresado en el Prefacio a Sobre el concepto de la DC, Fichte parece ahora convencido

${ }^{12}$ Fichte utiliza aquí la palabra Bedürfnis. La necesidad de la que se habla no remite únicamente al hecho de ser la única filosofía verdadera, sino que, tal como quedará claro a partir de la lectura de la Primera Introducción de 1797, la necesidad de la filosofía que, según Fichte, él mismo había sentido y que intentaba ahora despertar en sus oyentes y lectores, es en realidad un requisito, una necesidad práctica, una exigencia para la vida. Retomaremos esta idea hacia el final de este trabajo.

${ }^{13}$ GA, I/2, 253. Versión castellana: J.G. Fichte, Doctrina de la ciencia, trad. e intr. Juan Cruz Cruz, Aguilar, Buenos Aires, 1975, pp. 8-9. Es importante tener en cuenta que aquí Fichte no está utilizando la terminología kantiana, según la cual la forma de la «intuición interior» es el tiempo. Se trata, por el contrario, de una capacidad, una facultad que debe existir en aquellos que, según Fichte, pretendan comprender realmente su sistema. Esto es lo que en la Reseña del Enesidemo, y después, en la Segunda Introducción, Fichte denominará «intuición intelectual». 
de que su filosofía no es para todos. Los que quedan excluidos, aquellos a quienes Fichte solicita que no pierdan el tiempo con sus escritos, son aquellos hombres que carecen de aquella facultad que la DC indefectiblemente requiere: la libertad que hace posible lo que Fichte llama la intuición interior.

El Prólogo al Fundamento... presenta, pues, a la filosofía como el resultado de una necesidad y a la libertad como la condición de posibilidad en la cual esta necesidad parece fundarse. Ambos conceptos — necesidad y libertad — serán asimismo centrales en el desarrollo de la Primera Introducción a la DC y se revelarán, según creemos, como centrales en la dilucidación de la cuestión de qué es la filosofía, cómo ha de ser transmitida y quién es su destinatario.

2.

$\mathrm{Al}$ igual que en el Prólogo a los Fundamentos..., en la Advertencia Preliminar a la Primera Introducción a la DC Fichte asume su responsabilidad frente a la reacción negativa generada por su anterior publicación. Si bien afirma que esto no sucedió entre sus oyentes, que habían tenido la oportunidad de escuchar sus explicaciones y comprender así los pasajes oscuros, admite que ha expuesto su pensamiento de un modo confuso. En este sentido, el objetivo de esta nueva exposición de la DC es el de exponer su pensamiento del modo más claro posible. ${ }^{14}$

Pero también aquí Fichte traza un límite respecto de qué críticas está dispuesto a aceptar. Hace referencia entonces a aquellos lectores que, además de no comprender su pensamiento, lo han tomado como objeto de burlas e injurias, y han expresado repugnancia frente a sus ideas. ${ }^{15}$ "Criticar sin razones no significa nada, salvo que esta doctrina no agrada y esta afirmación, de nuevo, no tiene ninguna importancia», ${ }^{16}$ escribe. No se trata, según él, de si su pensamiento agra-

${ }^{14}$ Cf. GA I/4, 184.

${ }^{15}$ Cf. GA I/4, 184.

${ }^{16}$ GA I/4, 185. La versión castellana de la Primera Introducción a la DC que incluimos en este artículo es nuestra. Existen dos traducciones al castellano publicadas, que consultamos para este trabajo: Fichte, J.G., Primera y segunda introducción a la teoría de la ciencia, trad. J. Gaos, Madrid, Revista de Occidente, 1943, reimpr. Madrid, Sarpe, 1984 y Fichte, J.G., Introducciones a la Doctrina de la Ciencia, trad. M. Quintana Cabanas, Madrid, Tecnos, 1987. 
da o no, sino de si ha quedado demostrado. Es por eso que en esta Primera Introducción se propone responder a todas las posibles objeciones a las que su doctrina estaría expuesta. Pero, sospecha Fichte, incluso esto no será suficiente para un determinado tipo de lector. Por lo tanto, ve la necesidad de hacer una aclaración respecto del destinatario de su obra:

Escribo únicamente para aquellos, en quienes habita todavía el sentido interno para la certeza o la dubitabilidad, para la claridad o la confusión de su conocimiento, escribo para aquellos que atribuyen algún valor a la ciencia y a la convicción, y que son empujados por un vivo afán a buscarlas. Con aquellos que, como resultado de una larga servidumbre espiritual, se han perdido a sí mismos y que, consigo, han perdido el sentimiento de la propia convicción y su fe en la convicción de los otros, con aquellos que consideran una necedad que alguien busque independientemente la verdad, que no ven en las ciencias nada más que un modo cómodo de ganarse el pan, y que se estremecen ante cada ampliación de las mismas, como ante un nuevo trabajo, con aquellos para quienes ningún medio es infame en vistas a ocultar al corruptor de la empresa — con ellos no tengo nada que hacer. ${ }^{17}$

Retomando lo dicho en el Prólogo al Fundamento..., Fichte afirma que ciertos hombres no están capacitados para leer su escrito. Se trata de aquellos a quienes llama esclavos de espíritu, incrédulos que han perdido la capacidad de convencerse, hombres que ven en las ciencias un medio y no un fin y que desprecian a quienes se comprometen con la empresa de la búsqueda de la verdad. Respecto de ellos afirma, incluso, que le resultaría penoso que lo entiendan y expresa su deseo de que esta nota preliminar los confunda hasta tal punto que abandonen allí la lectura. Pues, según Fichte, «lo que en ellos ocupa la plaza del espíritu es arrastrado aquí y allá por su ciego furor interior» ${ }^{18}$.

La DC, en cambio, es para aquellos que poseen en sí mismos el criterio para distinguir lo claro de lo oscuro, lo verdadero de lo falso, para aquellos que son movidos por un impulso interior a buscar una convicción. Comenzamos a vislumbrar, pues, en qué sentido la filosofía se impondría como una necesidad: en tanto que

\footnotetext{
17 GA I/4, 185.

${ }^{18} \mathrm{GA}$ I/4, 185.
} 
es el resultado de ese impuso interior que mueve a ciertos hombres a buscar una verdad. Por el contario, aquellos que carecen de este impulso y que se abandonan a un ciego furor, no necesitan de la filosofía y, por ello, Fichte les pide que dejen sus libros de lado. Pues, como quedará claro a lo largo de la Primera Introducción, este afán, esta necesidad de buscar la verdad nace, surge, tiene su fundamento en esa libertad que la DC requiere como su condición indiscutible.

3.

Fichte no explicita, al comienzo de la Primera Introducción, que comenzará por la indagación acerca de qué es la filosofía; y en vez de brindar una definición de lo que él entiende por esta ciencia, una explicación de su concepto, Fichte inaugura el parágrafo primero de este texto con un imperativo: «Fíjate en ti mismo; aparta tu mirada de todo lo que te rodea y dirígela a tu interior ${ }^{19}$. Se trata, según él, de la primera exigencia que hace la filosofía a quien pretende ingresar en ella. La filosofía exige al aprendiz que realice una introspección, pues en la filosofía, escribe Fichte, «no se trata de nada que esté fuera de ti, sino únicamente de ti mismo» ${ }^{20}$.

Es precisamente el resultado de esta introspección, de este primer análisis de los contenidos de la propia conciencia, lo que conduce a Fichte a su definición de la filosofía como la ciencia que ha de indicar el fundamento de un determinado tipo de representaciones: aquellas determinaciones inmediatas de la conciencia que por no ser producto de la voluntad o la fantasía del sujeto que reflexiona, van acompañadas del sentimiento de la necesidad. Las representaciones que van acompañadas por el sentimiento de la libertad no plantean el problema de su fundamento, pues la propia voluntad libre es la explicación suficiente de por qué éstas son de tal o cual manera. El interrogante, en cambio, es planteado por aquellas representaciones cuyo fundamento no parece encontrarse en las facultades del sujeto que las piensa. Según Fichte, responder a la cuestión del fundamento del sistema de las representaciones acompañadas por el sentimiento de la necesidad y de ese mismo sentimiento es «la tarea de la filosofía ${ }^{21}$. Dado que el sistema de estas representaciones, tomado

\footnotetext{
${ }^{19}$ GA I/4, 186.

${ }^{20} \mathrm{GA} \mathrm{I} / 4,186$.
} 
en conjunto, es llamado por Fichte experiencia, la filosofía se presenta como aquella ciencia que debe indicar el fundamento de toda experiencia.

Contra aquellos que quisieran objetar que la filosofía es otra cosa, Fichte afirma que esto es exactamente lo que ha sido considerado como filosofía «siempre y por todos los conocedores ${ }^{22}$. Sin embargo, para evitar una discusión que considera ociosa, decide abandonar el nombre de filosofía y llamar a esta disciplina Doctrina de la ciencia.

El segundo parágrafo consiste en un análisis del concepto de fundamento, que acaba de revelarse como aquello que constituye el objeto de la filosofía. Fichte afirma que únicamente lo que se considera como contingente puede motivar la pregunta por cuál es su fundamento. La tarea de buscar el fundamento de una cosa contingente significa, según Fichte, «mostrar otra cosa, cuya determinidad [Bestimmtheit] permita comprender por qué lo fundado, entre las múltiples determinaciones [Bestimmungen] que podrían pertenecerle, tiene precisamente aquellas que tiene» ${ }^{23}$. De modo que lo fundante y lo fundado forzosamente pertenecen a ámbitos distintos: el fundamento, por el mero hecho de ser pensado como fundamento, sostiene Fichte, cae fuera de lo fundado y la relación entre ambos es de referencia mutua y, a la vez, de oposición.

Dado que la filosofía pretende exponer el fundamento de la experiencia, el objeto de la filosofía «se encuentra necesariamente fuera de toda experiencia» ${ }^{24}$. La tarea de la filosofía será, pues, la de elevarse sobre el sistema de las representaciones acompañadas del sentimiento de la necesidad, para buscar aquello que permita comprender por qué estas representaciones son como son. No aceptar esta definición de lo que es un fundamento, sostiene Fichte, equivale a negar la posibilidad de la filosofía misma, tal como aquí se entiende ${ }^{25}$.

Ahora bien, el parágrafo siguiente comienza planteando la paradoja implícita en la tarea de la filosofía así definida: el ser racional finito - y por lo tanto, también el filósofo- «no tiene nada fuera de la experiencia» ${ }^{26}$. La filosofía, en tanto exige

${ }^{21}$ GA I/4, 186.

${ }^{22}$ GA I/4, 187.

${ }^{23} \mathrm{GA} \mathrm{I} / 4,187$.

${ }^{24} \mathrm{GA} \mathrm{I} / 4,187$.

${ }^{25}$ Cf. GA I/4, 188. 
un ir más allá del ámbito de la experiencia, se presenta en principio como una tarea imposible. Sin embargo, existe una manera de sortear este aparente dilema.

Para elevarse por encima de la experiencia, el filósofo debe recurrir a su capacidad de abstracción, definida por Fichte como la acción de «separar lo que está unido en la experiencia mediante la libertad del pensar $\aleph^{27}$. Aquello que se encuentra unido en la experiencia son la cosa y la inteligencia. Así pues, al enfrentarse a la tarea de explicar el fundamento del sentimiento de necesidad que acompaña a un conjunto de sus representaciones, el filósofo descubre que la única vía que existe para llevar a cabo esta tarea requiere el ejercicio de su libertad.

La cosa, que se presenta como independiente del sujeto pensante, y la inteligencia, que conoce la cosa, son escindidas por el ente racional capaz de abstracción. Lo que en la experiencia es inseparable, se presenta ahora como dos polos diferentes, distinguibles uno del otro. $\mathrm{Al}$ abstraer de la experiencia y al elevarse sobre ella, el filósofo se encuentra con una disyuntiva.

Si el sujeto pensante abstrae de la cosa, sostiene Fichte, obtiene una inteligencia en sí. En cambio, si abstrae de la inteligencia, obtiene una cosa en sí. Ambas -inteligencia y cosa en sí- pueden ser postuladas como el fundamento del sistema de la experiencia. Si el filósofo propone que la inteligencia en sí es el fundamento buscado, entonces su posición se denomina idealismo. Si, al contrario, afirma que la cosa en sí permite explicar por qué determinadas representaciones vienen acompañadas del sentimiento de la necesidad, entonces su posición se denomina dogmatismo. La disyuntiva se revela, pues, como excluyente: dogmatismo e idealismo son las únicas dos formas que puede adoptar la filosofía.

4.

Fichte emprende, entonces, un análisis de la cosa en sí y el Yo en sí, únicos objetos posibles de la filosofía ${ }^{28}$.

${ }^{26} \mathrm{GA} \mathrm{I} / 4,188$.

${ }^{27} \mathrm{GA} \mathrm{I} / 4,188$.

${ }^{28}$ Fichte hace aquí un uso específico de la palabra objeto [Object]. Dice: «Llamamos al fundamento explicativo de la experiencia propuesto por una filosofía, el objeto de esta filosofía [das Object der Philosophie], puesto que éste parece existir únicamente por y para la misma» (GA I/4, 189). 
El objeto del Idealismo, el Yo en sí, es presentado como el resultado de un tomar conciencia de sí. Fichte propone al lector que se determine libremente a pensar una cosa determinada; luego, que abstraiga de lo pensado y se mire a sí mismo. Surge, entonces, el Yo como el contenido de una determinada representación. Las determinaciones particulares de ese Yo — el hecho de ser pensante y de estar pensando en tal o cual cosa- se revelan como el resultado de una autodeterminación. En efecto, el Yo pensante se determina a sí mismo a pensar una cosa en particular y se piensa a sí mismo como aquél que piensa eso determinado. Sin embargo, sostiene Fichte, esta experiencia enseña que «a mí mismo en sí no me hecho, sino que estoy obligado a pensarme como aquello que la autodeterminación ha de determinar ${ }^{29}$. De modo que el Yo se revela como algo cuya naturaleza o constitución particular depende de la inteligencia libre, pero cuya existencia debe ser siempre presupuesta.

El Yo en sí es, pues, según Fichte, «algo real, efectivamente en la conciencia ${ }^{30}$. No se trata de un objeto de la experiencia, pues no se presenta a la conciencia como algo exteriormente determinado, no se impone él mismo como necesario, sino que es el resultado de una autodeterminación del sujeto pensante que realiza el proceso de abstracción. "Sin esta determinación no es nada», escribe Fichte, «y sin ella ni siquiera es» ${ }^{31}$.

Tampoco la cosa en sí se presenta en la experiencia. No se trata de una representación acompañada por el sentimiento de la necesidad, sino que es un objeto producido completamente por la inteligencia libre. Y si bien el dogmático, tal como Fichte lo presenta, desea que la cosa en sí posea realidad, ésta se revela como una «mera invención» ${ }^{32}$. Su realidad únicamente podría ser establecida, si el dogmático llegara a mostrar que efectivamente la cosa en sí es el fundamento de toda la realidad. Pero hasta tanto esto no suceda, debe ser considerada como una construcción hipotética.

El resultado de este análisis es que el objeto del idealismo posee una clara ventaja sobre el del dogmatismo: el Yo en sí puede mostrarse en la conciencia; la

${ }^{29} \mathrm{GA} \mathrm{I} / 4,190$.

${ }^{30} \mathrm{GA} \mathrm{I} / 4,190$.

${ }^{31} \mathrm{GA} \mathrm{I} / 4,190$.

${ }^{32} \mathrm{GA} \mathrm{I} / 4,190$. 
cosa en sí, en cambio, es una mera invención del sujeto pensante. Esto, sin embargo, no excluye aún al dogmatismo como filosofía posible. En efecto, Fichte sostiene que esta distinción no apunta a descalificar al dogmatismo, sino simplemente a señalar su diferencia con el idealismo ${ }^{33}$.

El cuarto parágrafo concluye con una consideración acerca de posibles objeciones a este análisis. Respecto de que la cosa en sí sea una mera invención, Fichte afirma que únicamente alguien que no ha entendido bien su explicación podría objetar algo. Respecto de su caracterización del Yo en sí, Fichte atiende a la posibilidad de que alguien niegue la conciencia de sí inmediata en una acción libre del espíritu. A esta objeción, responde:

Esta autoconciencia no se impone ni viene de suyo; hay que actuar efectivamente de un modo libre, y luego abstraer del objeto y únicamente fijarse en sí mismo. Nadie puede ser obligado a hacer esto, y aunque se pretenda hacerlo, es imposible saber si procede rectamente y como se requiere. En una palabra, esta conciencia no puede comprobarse en nadie; cada uno debe producirla en sí mismo por medio de su libertad. ${ }^{34}$

Nuevamente, reaparece la idea de que la operación espiritual sobre la que se funda la filosofía no se da en la actitud natural de los seres humanos ni puede ser impuesta desde el exterior. Tampoco puede ser observada en otra persona, ni podría verificarse que un sujeto la esté llevando a cabo correctamente. La autoconciencia que es la primera exigencia de la filosofía sólo puede darse en el interior de cada sujeto pensante, como resultado del ejercicio de su libertad.

El análisis de la noción de filosofía y de sus posibles objetos que Fichte desarrolla a lo largo de los cuatro primeros parágrafos de esta Primera Introducción, arroja dos consecuencias fundamentales. En primer lugar, la necesidad de que el filósofo posea la capacidad de ser consciente de sí mismo y de ejercer la abstracción - capacidad que se fundamenta en su libertad. Se trata, pues, de lo que Fichte había adelantado en el Prólogo al Fundamento...: aquella «facultad de libertad de la intuición interna» de la que los esclavos de espíritu carecerían por

\footnotetext{
${ }^{33}$ Cf. GA I/4, 190.

${ }^{34}$ GA I/4, 191.
} 
completo $^{35}$. La filosofía, entendida como una tarea, como un camino de búsqueda de un fundamento que requiere el ejercicio de una determinada facultad, sólo es posible si el filósofo es capaz de ejercer su libertad. No hay modo de comprobar exteriormente si el sujeto lo realiza o no, cada uno se transforma interiormente en el único testigo y juez de su propio actuar libre.

En segundo lugar, este análisis de la noción misma de filosofía adoptada por Fichte conduce a afirmar que «sólo estos dos sistemas filosóficos [el dogmatismo y el idealismo] son posibles» ${ }^{36}$. Sólo existen dos posibilidades si, al querer exponer el fundamento de la experiencia, se procede de un modo consecuente: o bien el sentimiento de la necesidad proviene de una cosa en sí o bien proviene de una inteligencia en sí. Así pues, si la DC había comenzado imponiendo al aprendiz de filósofo la exigencia de fijarse en sí mismo, el resultado de esta introspección no es un cúmulo de conocimientos, un conjunto de conceptos, sino que es la urgencia de tomar una decisión: o dogmatismo o idealismo.

\section{5.}

La relación entre dogmatismo e idealismo se revela como compleja. "Ninguno de estos dos sistemas puede refutar directamente a su opuesto", sostiene Fichte, "pues el desacuerdo entre ellos es un desacuerdo acerca del primer principio, que no puede derivarse de otro ${ }^{37}$. No poseen ningún punto en común, por lo que la discusión entre ellos se encuentra vedada desde el comienzo. Según el idealista, todo lo que se presenta en la conciencia encuentra su fundamento en la inteligencia libremente actuante. Según el dogmático, este fundamento reside en una cosa en sí, incluso aquellas representaciones que muestran al sujeto como libre.

La libertad se transforma para el dogmático en una ilusión generada por la cosa en sí que se combina con la ignorancia del sujeto que no puede remitir esas representaciones a su verdadera causa y las atribuye a su propia espontaneidad ${ }^{38}$.

\footnotetext{
${ }^{35}$ Véase más arriba el apartado 1.

${ }^{36} \mathrm{GA} \mathrm{I} / 4,188$.

${ }^{37} \mathrm{GA} \mathrm{I} / 4,191$.

${ }^{38}$ Cf. GA I/4, 192.
} 
Por eso Fichte establece que «todo dogmático consecuente es necesariamente fatalista ${ }^{39}$. El Yo no es para él algo independiente, sino un resultado de una serie causal, siempre sujeto a la influencia y la determinación por parte de las cosas: un «accidente del mundo» ${ }^{40}$, dice Fichte. Y por eso, además de fatalista, el dogmático consecuente debe ser también materialista ${ }^{41}$.

Dogmatismo e idealismo son dos sistemas absolutamente incompatibles y no pueden refutarse mutuamente. Su combinación en un único sistema sería totalmente inconsecuente ${ }^{42}$. La pregunta a la que conduce este análisis es, por lo tanto, ¿qué puede mover a un hombre, que comprende las diferencias entre ambos sistemas, a preferir uno al otro? ¿Qué impulsa a afirmar la independencia del Yo, sacrificando la independencia de la cosa o, a la inversa, a sacrificar la independencia del Yo y a postular la cosa en sí como el fundamento último de toda experiencia? Frente a la aparente arbitrariedad de esta decisión, Fichte se pregunta finalmente por qué no se ha difundido el escepticismo, que representaría la renuncia a tener que optar.

En primer lugar, contra la posibilidad de la instauración general del escepticismo, Fichte indica que el filósofo, como todo hombre, se encuentra en la situación de tener que aceptar que no puede sino representarse que él es libre y que existen las cosas fuera de él. Sin embargo, le es imposible permanecer en este pensamiento: «El pensamiento de la mera representación es sólo medio pensamiento, un fragmento inacabado de pensamiento» ${ }^{43}$. La pregunta por el fundamento de las representaciones es, pues, inevitable. E inevitablemente también la respuesta debe señalar un único primer principio: o bien la inteligencia es lo independiente y de ella depende el sistema de las representaciones, o bien lo es la cosa

${ }^{39}$ GA I/4, 192. La caracterización del spinozismo como un determinismo que, por ser consecuente, conduce al fatalismo se encuentra en la interpretación que Jacobi expone de esta doctrina en sus Cartas sobre la doctrina de Spinoza al Sr. Moses Mendelssohn (véase Jacobi, Friedrich Heinrich, Über die Lehre des Spinoza in Briefen an den Herrn Moses Mendelssohn, Breslau, 1785. Segunda edición: 1789). La caracterización del spinozismo como dogmatismo puede rastrearse en el artículo de Kant, publicado en octubre de 1786, «¿Qué significa orientarse en el pensamiento?» (véase Kant, I., "Was heißt: sich im Denken Orientieren?», Berlinische Monatsschrift, octubre de 1786).

${ }^{40}$ GA I/4, 192.

${ }^{41}$ Cf. GA I/4, 192.

${ }^{42}$ Cf. GA I/4, 192.

${ }^{43}$ GA I/4, 193. 
en sí. ¿Cómo elegir? ¿Qué es lo que motiva que un hombre se incline por uno o por otro? La respuesta de Fichte es la siguiente:

Ningún fundamento para la decisión puede provenir de la razón; pues no se trata aquí del enlace de un eslabón en la serie, para lo cual los fundamentos racionales alcanzan, sino que se trata del comienzo de toda la serie, el cual, en tanto que es absolutamente el primer acto, depende únicamente de la libertad del pensar. Así pues, este acto es determinado por el libre arbitrio, y como la resolución del libre arbitrio debe tener un fundamento, éste es determinado por inclinación e interés [Neigung und Interesse]. ${ }^{44}$

Dogmáticos e idealistas se distinguen, en última instancia, por lo que Fichte llama su inclinación, su interés - y que de ningún modo pueden ser entendidos como una inclinación o un interés utilitarista, calculador. Cuál sea el interés que guía las decisiones libres de los hombres depende, según él, de qué clase de hombre se es...

De modo que nuevamente aquí, en el transcurso del análisis de su noción de filosofía, volvemos a encontrar una clasificación de los seres humanos según algo que, aparentemente, ellos mismos difícilmente pueden torcer y que sin embargo es lo que determina si son o no aptos para la que se revelará como la verdadera filosofia.

Fichte sostiene que existen "dos grados [Stufen] de la Humanidad» y que, hasta tanto el grado superior no haya sido conquistado por todos, los seres humanos se dividen en dos especies. La primera especie es presentada del siguiente modo:

Unos, que aún no se han elevado al pleno sentimiento de su libertad y de su absoluta autonomía, se encuentran a sí mismos únicamente en el representarse las cosas; poseen únicamente esta conciencia de sí dispersa, adherida a los objetos, que ha de ser derivada a partir su multiplicidad. Su imagen les es arrojada sólo a través de las cosas, como a través de un espejo; si éstas les fueran arrebatadas, perderían junto con ellas su yo. En vistas a sí mismos,

${ }^{44} \mathrm{GA}, \mathrm{I} / 4,194$. 
no pueden renunciar a la fe en la independencia de las cosas, pues ellos mismos existen sólo con ellas. ${ }^{45}$

Ésta es la esencia del dogmático, que se considera a sí mismo un producto del mundo en el que se encuentra arrojado. El principio sobre el cual construyen su filosofía es, pues, lo que Fichte llama «la fe en las cosas», en la que se sostiene la creencia en su propio yo. Esta actitud, este interés, descansa en el hecho de que el dogmático no experimenta el sentimiento de su libertad: no se siente independiente del mundo exterior. Este es, precisamente, el punto en el cual el idealista se distingue de él. Dice Fichte:

Quien llega, sin embargo, a ser consciente de su autonomía e independencia de todo lo que existe fuera de él —y esto sólo se logra, cuando uno hace de sí mismo algo por sí mismo, independientemente de todo-, no necesita de las cosas como el sustento de su yo, y no puede servirse de ellas, pues ellas anulan aquella autonomía y la transforman en mera apariencia. El yo, que él posee y que a él le interesa, anula aquella fe en las cosas. Por su inclinación, él cree en su autonomía y la abraza apasionadamente. Su fe en sí mismo es inmediata. ${ }^{46}$

Se confirma, así, que la razón especulativa no juega aquí ningún papel. El hecho de ser dogmático o idealista se dirime plenamente en el ámbito del interés, de la inclinación, del afecto, de la fe. Fe en sí mismo como el fundamento primero e independiente de toda la experiencia; fe en las cosas, como aquello originario de lo cual depende incluso el propio yo. Lo que distingue al dogmático del idealista es que el primero no puede sentirse libre. El segundo, en cambio, experimenta su libertad en su propio actuar. $Y$ en este sentido se comprende plenamente el párrafo, tan citado, que Fichte incluye hacia el final del quinto parágrafo de esta Introducción:

Qué clase de filosofía se elige depende, por lo tanto, de qué clase de ser humano se es. Pues un sistema filosófico no es como un ajuar muerto, que uno pueda rechazar o adoptar según nos convenga, sino que es animado por el alma del ser humano que lo posee. ${ }^{47}$

\footnotetext{
${ }^{45} \mathrm{GA}, \mathrm{I} / 4,194$.

${ }^{46}$ GA I/4, 194-5.

${ }^{47}$ GA I/4, 195.
} 
La conclusión que Fichte extrae de aquí, y que se sigue directamente de su noción de filosofía, es central para la cuestión del destinatario de la DC:

Un carácter flojo [schlaffer] por naturaleza, o que ha sido debilitado y doblegado por la servidumbre de espíritu, el lujo refinado y la vanidad, jamás podrá elevarse al idealismo. [...] Para ser filósofo — si el idealismo llegara a probarse como la única filosofía verdadera-, para ser filósofo hay que haber nacido, hay que haber sido educado y educarse a sí mismo para ello. Pero es imposible ser hecho para ello mediante ningún arte humano. ${ }^{48}$

Si bien el idealista lograría elevarse sobre el dogmatismo, pues la conquista de esta conciencia de la propia autonomía frente al mundo exterior, que caracteriza la esencia del idealista y determina su elección del principio fundamental de toda experiencia, es según Fichte el resultado de haber pasado por el estado dogmático y de haber reconocido su falsedad ${ }^{49}$, el dogmático parece condenado a permanecer siempre aferrado a las cosas... y por lo tanto, condenado a permanecer siempre al margen de la verdadera filosofía. Su incapacidad de ser consciente de su propia autonomía reside en el hecho de que o bien posee naturalmente un carácter débil, flojo o bien su carácter ha sido entumecido y el sujeto se ha transformado en un esclavo de espíritu.

Se trata de aquellos a quienes Fichte pide, en la Advertencia Preliminar, que ni siquiera lean su obra. La incapacidad de estos hombres de comprender sus palabras reside en el hecho de que carecen de la facultad que la DC requiere: la facultad de la intuición interna de su propia libertad. Ahora bien, el problema al que nos enfrentamos aquí es que, según Fichte, «los filósofos modernos son en su totalidad dogmáticos y están firmemente resueltos a seguir siéndolo.» ${ }^{50}$

¿A quién se dirige entonces la DC, si para ser filósofo hay que haber nacido, ser educado y educarse para ello? ¿Cómo es posible que entre los dogmáticos se produzca el surgimiento de un filósofo idealista? La respuesta explícita de Fichte, no carente de ambigüedad, es que si la DC encontrará «pocos prosélitos entre

${ }^{48} \mathrm{GA} \mathrm{I} / 4,195$.

${ }^{49}$ «... al idealismo se pasa, si no a través del propio dogmatismo, al menos a través de la inclinación sentimental a él» (GA I/4, 195).

${ }^{50} \mathrm{GA} \mathrm{I} / 4,191$. 
los hombres ya hechos», sus esperanzas están más bien depositadas en «el mundo juvenil». Esto se debe a que, a diferencia de los viejos pensadores, las mentes jóvenes poseen una fuerza que "no ha sucumbido aún en la flojera de la época» ${ }^{51}$.

Puede ser que Fichte haya depositado la mayor parte de sus esperanzas en los estudiantes de la Universidad de Jena... sin embargo, el hecho de que escriba una Segunda Introducción destinada a aquellos que ya poseen un sistema filosófico, hace pensar que guarda aún una pequeña expectativa respecto de la posibilidad de convertir al dogmático en idealista, es decir, de impulsar su elevación al grado superior de la humanidad.

6.

Efectivamente, los últimos parágrafos de la Primera Introducción están destinados a mostrar las ventajas del idealismo y las carencias del dogmatismo, de modo que el primero se afirme ante los ojos del lector como la única verdadera filosofía. Ahora bien, si Fichte hablara únicamente a aquellos lectores que ya han nacido idealistas, estos parágrafos deberían ser declarados innecesarios.

Fichte dedica el sexto parágrafo a poner en evidencia que el dogmatismo no puede explicar la representación. Mientras que la inteligencia se desdobla en dos series, la real del ser y la ideal del ver, la cosa, en cambio, que no es para sí, presenta una única dimensión, la del mero $\operatorname{ser}^{52}$. Así, dado que el principio del dogmático reside simplemente en un ser, le es imposible mostrar el paso del ser al representar. Intenta explicar la inteligencia mediante el principio de causalidad, pero éste únicamente atañe a la serie real. El resultado es que el dogmático da "un enorme salto a un mundo totalmente ajeno a su principio» ${ }^{53}$ e intenta ocultarlo mediante explicaciones y palabras huecas que no brindan una verdadera explicación.

La cosa en sí postulada como principio no logra explicar lo que la filosofía se propone explicar. Fichte concluye, pues, que «el dogmatismo $[. .$.$] no es una$

${ }^{51}$ GA I/4, 195.

${ }^{52}$ Cf. GA I/4, 196.

${ }^{53} \mathrm{GA} \mathrm{I} / 4,197$. 
filosofía, sino sólo una impotente aseveración y afirmación». ${ }^{54}$ Se sigue de allí que «sólo queda el idealismo como la única filosofía posible.»" ${ }^{55}$

Ahora bien, volviendo sobre el aparente círculo en el que Fichte parece haber caído en el parágrafo anterior - en conexión con las dificultades de que la DC sea aceptada por los hombres ya formados- nuevamente se hace en este punto explícita la complejidad que encierra la posibilidad de que un dogmático devenga idealista:

Se necesita ya un cierto grado de autonomía y libertad de espíritu, para comprender la esencia de la inteligencia, tal como la hemos expuesto, en la cual se fundamenta toda nuestra refutación del dogmatismo. Muchos no han logrado ir con su pensamiento más allá de la comprensión de la serie simple del mecanismo natural. [...] Así pues, no se puede refutar al dogmático mediante la demostración expuesta, a pesar de su claridad; pues es imposible convencerlo de ella, ya que carece de la facultad con que sus premisas deben ser aprehendidas. ${ }^{56}$

Pero si este pasaje parece confirmar que el dogmático está condenado de por vida al dogmatismo, el último parágrafo de esta Introducción parece brindar nuevamente ciertas esperanzas.

Fichte emprende allí el análisis del principio del idealismo: el actuar de la inteligencia. Esta inteligencia, que no es un ente —ni siquiera un «ente activo»— sino una pura actividad absoluta, se revela como actuando de un modo determinado, según leyes que se siguen de su propia esencia. Este modo determinado de actuar de la inteligencia es lo que garantiza que de su actividad se deduzcan representaciones determinadas, las cosas que, en su conjunto, constituyen el mundo. Es gracias a estas leyes necesarias de la inteligencia que se explica el sentimiento de necesidad que acompaña a las representaciones de la conciencia: la inteligencia «siente en su actuar los límites de su propia esencia», ${ }^{57}$ escribe Fichte.

\footnotetext{
${ }^{54} \mathrm{GA} \mathrm{I} / 4,198$.

55 GA I/4, 198.

${ }^{56}$ GA I/4, 198-9.

${ }^{57} \mathrm{GA} I / 4,200$.
} 
Se extrae de aquí un método: el idealismo crítico o trascendental integral —el de la $\mathrm{DC}^{58}$ — procede del siguiente modo:

El idealismo crítico parte de una única ley fundamental de la razón, que muestra inmediatamente en la conciencia. A partir de allí, procede de la siguiente manera. Exige al oyente o al lector, pensar con libertad un concepto determinado; si lo hace, encontrará que se ve obligado a proceder de cierta manera. Aquí hay que distinguir entre dos cosas: el acto de pensar exigido, que es llevado a cabo por la libertad —y aquél que no lo lleva a cabo, no ve nada de lo que enseña la Doctrina de la ciencia- y la manera necesaria como hay que llevar a cabo ese acto, la cual está fundada en la naturaleza de la inteligencia y no depende del libre arbitrio; es algo necesario, que sin embargo sólo surge en y con una acción libre; algo encontrado, siendo el hecho de encontrarlo condicionado por la libertad. ${ }^{59}$

A diferencia del dogmatismo, que afirma impotentemente su primer principio, sin lograr exponer su realidad en la conciencia, exigiendo de su interlocutor que acepte como fundamento de la experiencia una mera invención, el idealismo integral, que es la única filosofía verdadera, «muestra en la conciencia inmediata lo que él afirma» ${ }^{60}$.

Es por esto que, frente a los dos caminos posibles en los que la filosofía se bifurca, «el pensador resuelto", afirma Fichte, siempre se decidirá por el idealismo. Y si bien el dogmático podría ser difícil, incluso imposible de convertir, el

\footnotetext{
${ }^{58}$ Fichte distingue entre un «idealismo trascendente», que deduce del actuar libre y sin ley de la inteligencia las representaciones determinadas y que se revela como contradictorio, pues de un actuar indeterminado — en el sentido de actuar sin ley — no puede deducirse nada determinado (cf. GA I/4, 201); y un «idealismo crítico o trascendental». Dentro de este último, distingue a su vez dos modos de proceder: un proceder según el cual se extraen las leyes del pensar tal como se aplican inmediatamente a los objetos y afirma que mediante éstas se determinan los objetos. Fichte critica este proceder, que permanece en el grado inferior de las leyes, que él llama "categorías», preguntándose de dónde saca este idealista estas leyes. No logra explicar por qué la inteligencia debe actuar de este modo... y por lo tanto, no logra explicar cómo es que surge el objeto. Pues únicamente explica las propiedades de la cosa, pero no de dónde proceden en tanto que cosas, no logra explicar el origen de su «materia» (cf. GA I/4, 201-203). El segundo proceder, el que describiremos a continuación, sí da respuesta a estas cuestiones y es el verdadero proceder de la DC.

${ }^{59} \mathrm{GA} \mathrm{I} / 4,204$.

${ }^{60}$ GA I/4, 205.
} 
idealismo presenta la misma ventaja en cuanto a su irrefutabilidad. Así, al menos, lo afirma la oración final del texto:

Demostraciones de la imposibilidad de una cosa que es realizada y en parte ya ha sido realizada, son tan solo ridículas. Hay que atenerse únicamente a la ejecución y comprobar si produce lo que ha prometido. ${ }^{61}$

La filosofía se revela, desde la primera hasta la última palabra de esta Primera Introducción, como una tarea a realizar que exige únicamente una cosa: actuar, ser libre, ser verdaderamente un filósofo.

7.

Digamos para concluir que es en tanto que la filosofía exige el ejercicio de la propia libertad, que ésta se transforma, como quería Fichte, en una necesidad para los seres humanos.

Todos necesitamos ir más allá de la mera representación y encontrar su fundamento... pero no cualquier fundamento satisface a aquél que se experimenta como autónomo al realizar el acto de introspección y de abstracción que la búsqueda de ese fundamento requiere. Sólo el actuar de la inteligencia según sus propias leyes permite explicar el sentimiento de la necesidad que acompaña a las representaciones que llamamos experiencia. Sólo ese Yo en sí, abstraído de lo que el yo piensa, es el último fundamento de todo lo que aparece como real y efectivo al Yo. Y, tal como Fichte lo adelantaba en el primer parágrafo, sólo de eso trata la filosofía.

Ahora bien, esta necesidad de postular un fundamento satisfactorio no consiste en una mera necesidad teórica, como si se tratara de un problema matemático que el filósofo debe resolver para poner su mente a descansar. En una nota al pie de la Segunda Introducción ${ }^{62}$, Fichte explicita en qué medida el idealismo, en tanto que posición especulativa, sólo existe para hacer comprensible la posi-

\footnotetext{
${ }^{61}$ GA I/4, 208.

${ }^{62}$ Cf. GA I/4, 210.
} 
ción de la vida: ese realismo que se impone a todos al momento de actuar. En este sentido, la finalidad de una filosofía es la deducción de una verdad objetiva, pero también la de aclarar el ámbito de la libertad, de las acciones humanas. El filósofo, que afirma al Yo como principio originario, se encuentra, pues, al servicio de ese mismo Yo, que afirma que, tan cierto como que él mismo existe, existen fuera de él cosas y un mundo que son independientes de él. El filósofo se limita, pues, a explicar, a dar un fundamento inteligible para aquello que el yo experimenta inmediatamente como una verdad indubitable.

La filosofía es una necesidad, un requisito para comprender la vida. $Y$, tal como vimos, el único requisito para ser un verdadero filósofo es, según Fichte, la capacidad de ejercer la propia libertad... y tal vez en este sentido pueda pensarse que, si bien el aprendiz de filósofo ya debe contar con esa capacidad, la DC, en tanto que es un puro proceder, en tanto que exhibe en la conciencia del lector/oyente lo que ella quiere afirmar, pone en evidencia que, para el dogmático que se encuentra en una situación de esclavitud espiritual, pero que está dispuesto a tomar consciencia de su penosa situación y que no está empeñado en permanecer en ella, la verdadera filosofía puede ser también un camino de educación y de liberación. 
